\title{
35. SUMMARY OF MAGNETIC OBSERVATIONS, LEG 49
}

\author{
M. Steiner, University of Wyoming, Laramie, Wyoming; \\ R. Day, University of California, Santa Barbara, California; \\ K. Kobayashi, Ocean Research Institute, University of Tokyo, Japan \\ and \\ A. Faller, University of Leeds, Leeds, England
}

The preceding chapters have described diverse magnetic studies of the Leg 49 basalt samples. In this chapter, we will summarize the combined observations on paleomagnetism, rock magnetism, magnetochemistry, and opaque microscopy of these samples. Many of our conclusions are in agreement with previous studies of oceanic basalts (see Legs 34,37 , and 45 , for example). In general, we find low intensities (about 5 $\times 10^{-3} \mathrm{G}$ ) of remanence (NRM), implying that the source of the magnetic anomaly patterns of the oceans has to be much deeper than our deepest penetrations into basalt (maximum $=$ $230 \mathrm{~m}$ ). This is the same conclusion reached by Ryall et al. (1977) from a study of nearly 600 meters of oceanic crust cored on Leg 37.

As has been observed in most previous studies of the magnetization of oceanic crust, the remanence dominates the magnetization; all but a very few samples show low susceptibility $(\chi)$, and thus high $Q$ values $(\mathrm{Jn} / \chi \mathrm{H})$. Those samples showing high $\chi$ have larger grain sizes, in agreement with observations by Johnson and Hall (1976) that grain size and associated permeability of the unit controls the degree of oxidation. (Fine grain size accompanies a high permeability to seawater, as in pillow basalts; coarse-grained units have low permeability to seawater and are oxidized less.)

All our samples show a low degree of low-temperature oxidation (low $z$ values), even though they range in age from just less than $1 \mathrm{~m}$.y. to almost $40 \mathrm{~m}$.y. The least oxidized are still oxidized to some extent, however. Even the youngest samples ever recovered by DSDP (the 0.95-m.y. samples of Hole 411) show that appreciable oxidation had occurred by 1 m.y. ago, in agreement with Macdonald's (1977) deeptowed magnetometer observations within the median valley at $37^{\circ} \mathrm{N}$. His data suggest that much of the low-temperature oxidation that reduced the remanence occurred by about 0.6 m.y. ago. He observed that magnetization intensity dropped very sharply for the first 0.2 to 0.3 million years, after which the decrease was more gradual (fig. 11 of Macdonald, 1977; also Johnson and Atwater, 1977). Hole 411 samples (about $0.95 \mathrm{~m} . \mathrm{y}$.) show the same NRM intensities as those of all of our other sites, indicating that by at least $0.95 \mathrm{~m} . \mathrm{y}$. ago, substantial oxidation and concomitant remanence reduction had occurred.

Our studies show that samples of the oceanic crust comprise a roughly bimodal population, slightly oxidized and relatively more oxidized. Curie temperature $\left(T_{c}\right)$ curves are generally either reversible with a low $\mathrm{T}_{\mathrm{c}}\left(125^{\circ}\right.$ to $\left.210^{\circ} \mathrm{C}\right)$ or irreversible with a higher $\mathrm{T}_{\mathrm{c}}\left(>210^{\circ}\right.$ to $430^{\circ} \mathrm{C}$; Kobayashi et al., this volume). Similarly, the properties of saturation magnetization $\left(\mathrm{J}_{\mathrm{s}}\right)$, saturation remanence $\left(\mathrm{J}_{\mathrm{rs}}\right)$, bulk coercivity $\left(\mathrm{H}_{\mathrm{c}}\right)$, remanent coercivity $\left(\mathrm{H}_{\mathrm{rc}}\right)$, and susceptibility $(\chi)$ are divided into a more oxidized (low $\mathrm{J}_{\mathrm{s}}, \mathrm{J}_{\mathrm{rs}}$, and $\chi$, high $\mathrm{H}_{\mathrm{c}}$ and
$\mathrm{H}_{\mathrm{rc}}, \mathrm{H}_{\mathrm{c}}>100 \mathrm{OE}$ ) and a less oxidized (higher $\mathrm{J}_{\mathrm{s}}, \mathrm{J}_{\mathrm{rs}}$, and $\chi$, lower $\mathrm{H}_{c}$ and $\mathrm{H}_{\mathrm{rc}}, \mathrm{H}_{\mathrm{c}}>100 \mathrm{OE}$ ) (Day et al., this volume). The overall effect of oxidation, as has been noted by Irving (1970) at $45^{\circ} \mathrm{N}$ and Ryall et al. (1977) at $37^{\circ} \mathrm{N}$ and many others, is to reduce the intensity of the remanence, raise the coercivity and Curie temperature, and reduce the susceptibility, thereby producing a more stable, lowered intensity magnetization. However, as illustrated by the $0.95-\mathrm{m}$.y.-old samples, the remanence reduction is very fast, after which it changes more slowly, while the other properties continue to change noticeably with increasing oxidation.

The coring program on Leg 49 was oriented toward observing possible variations within the oceanic crust, variations along the spreading ridge, with depth, and in time (perpendicular to the ridge). In general, our measurements show no differences along the ridge. We expected a trend attributable to the presence of the Icelandic "plume," and apparent trends found by Schilling (1973). There is no evidence of an increase in titanium content in the oxidized titanomagnetites (hereafter referred to as "titanomagnetites"), in either Curie temperature or microprobe $x$ values of the least oxidized samples, between the FAMOUS area $\left(37^{\circ} \mathrm{N}\right)$ and the sites on the Reykjanes Ridge $\left(62^{\circ} \mathrm{N}\right)$. However, the lack of a trend is in agreement with an apparent preferential formation of titanomagnetite with $x \approx 0.65$ in all oceanic basalts (Johnson and Hall, in press).

No systematic variation of degree of oxidation appears to occur with either age or depth. Both more and less oxidized samples alternate downhole in all holes except 410, 410A, and $412 \mathrm{~A}$. Hole $412 \mathrm{~A}$ samples ( 2 m.y.) are only slightly oxidized, and less oxidized than the younger Hole 411 samples. Samples from the Site 410 holes ( $10 \mathrm{~m}$.y.) are the most oxidized of the Leg 49 basalts - more so than the nearly 20 m.y. older Hole 407 basalts ( 38 m.y.). As will be discussed later, this is probably related to the finer grain size, and to somewhat different conditions of formation of the upper basement units in this area. These inconsistencies show the danger of generalizing from single sites within the oceanic crust. But overall, oxidation in the basalts of this study does not seem to be related to age or depth, suggesting that degree of oxidation depends on hydrothermal circulation within the oceanic crust. No systematic variation of intensity of natural remanent magnetization, either with age, with depth, or spatially along the ridge, was found (except for that to be expected from the greater magnetizing field of higher latitudes). Variations in the rock magnetic properties of saturation magnetization, saturation remanence, susceptibility, coercive force, and remanent coercive force also were not systematic in any of these ways. They seem to be related primarily to the degree of oxidation that has occurred, and 
thus also vary nonsystematically with depth and age.

Despite the nonsystematics of oxidation described, however, there is a general tendency for oxidation to be somewhat less in the younger rocks than in the older ones. Lower Curie temperatures, more reversible Curie curves, and optically fresher titanomagnetite (see Figure 1) near the ridge, particularly at Site 411 and Hole 412A, indicate somewhat less oxidation near the ridge relative to the rest of the sites. However, samples from Sites 407 and 409, 35 m.y. difference in age (Site $409=2.3$ m.y.), are extremely similar in their degree of oxidation. Penetration at both sites was in excess of 100 meters, so the results are representative.

The $x$-values seem to be uniform in time ( 1 to $40 \mathrm{~m}$.y.), and in space $\left(62^{\circ} \mathrm{N}\right.$ to $\left.37^{\circ} \mathrm{N}\right)$ relative to the hypothetical Icelandic hot spot. The average of $x=0.66$ (range $=0.50$ $<x<0.86$ ) is in agreement with the observation by Johnson and Hall (1978) that oceanic basalts tend strongly to have $x$ values around 0.62 . We noted a tendency for more oxidized samples (higher $\mathrm{T}_{\mathrm{c}}$ ) to have higher $x$ values (see Kobayashi et al., this volume). This may be explained by the hypothesis (Johnson and Hall, in press) that iron is progressively removed from the lattice during oxidation, to form the red staining of the silicates. Iron and titanium both migrate from the lattice, but iron migrates faster than titanium. Preferential removal of iron would relatively enrich titanium, giving more oxidized basalts higher $x$ values.

The lack of overall trends in magnetic properties at these seven sites suggests a general uniformity of the oceanic crust in space and time (at least to $40 \mathrm{~m}$.y. age). Some unique spots exist. Site 410 is unusual. Here, at two holes spaced 110 meters apart, basalts were cored which have (relative to the other sites) much finer grained titanomagnetites, higher Curie temperatures (all irreversible), red-stained silicates, high and uniform median destructive fields (MDF), high coercive force, low saturation remanence, and low susceptibility, all indicating a more highly oxidized titanomagnetite. One of the two holes drilled (410) recovered only brecciated basalt chunks in a limestone matrix. The other hole (410A) appeared to penetrate continuous flows of pillow basalt which had, however, magnetic properties identical to the breccia. Even though a breccia, the inclinations of the breccia pieces were predominantly positive, in agreement with the sign of the anomaly. Furthermore, smaller basalt pieces (smaller than the diameter of the core) of the breccia seemed even more uniform in their properties, and more oxidized, than larger chunks (larger than the diameter of a DSDP core). This is suggested by the partial consistency of inclinations of individual small basalt pieces in near proximity to one another (Figure 2), although there is no consistency from one group of small pieces to another group (see Figure 2 and Site Report, Site 410). Tests for randomness (discussed in the Site Report chapter) indicated that the directions are not small basalt pieces are more resistant to alternating field demagnetization (Figure 3), and their Curie temperatures are more uniform than those of the larger basalt pieces (Figure 1). The limestone matrix of the breccia also has a coherent positive remanence similar to the large basalt pieces.

All the basalt chunks of the breccia are highly oxidized. The higher (and irreversible) Curie temperatures, higher coercive force, and low saturation magnetization all indicate a more oxidized basalt than was seen at the other Leg 49 sites. Opaque microscopy showed a very fine grain size $(0.25$ to $0.5 \mu \mathrm{m})$, brown rims on all the titanomagnetite, a gray to gray-white color of the titanomagnetite, and red staining of the silicate matrix in all samples.

Our results suggest the following: (1) The basement formations in Holes 410 and $410 \mathrm{~A}$ are related. Both sites are very fine grained, are microscopically similar, and have similar Curie temperatures, susceptibility, and hysteresis properties, indicative of a moderately high degree of oxidation. (2) The breccia from Hole 410 probably formed at elevated temperatures. This is suggested by the overall lack of randomness of breccia pieces, the fairly oxidized state of the titanomagnetite, and the coherence of the matrix remanence and its similarity in direction and stability to that of the basalt pieces. The fine grain size of both holes suggests quenching. The high state of oxidation and the matrix remanence suggest that the basement was hot during and after brecciation. Basalt pieces from the breccia of Hole 410 may be even slightly more oxidized than the pillows of Hole 410A, as suggested by their slightly higher Curie temperatures. The properties of the two holes are consistent with having drilled the brecciated tip of a series of flows in Hole 410 and slightly farther away from the tip in coherent (quenched) pillow basalts in Hole $410 \mathrm{~A}$, although the thickness of the breccia $(20 \mathrm{~m})$ strains this hypothesis. Twenty meters of breccia would require equally deep sediment ponds, a feature which has rarely been found on presently active ridge crests. Thus, perhaps brecciation took place as the blocks were transported up and out of the median valley, but brecciation'occurring while still in the hot regime of the median valley, is a more tenable explanation (H.P. Johnson, personal communication).

Sites 407 and 409 were interesting in that magnetic divisions could be seen, both in the paleomagnetic and rock magnetic data. At Site 407, three magnetic units were distinguished on the basis of paleomagnetic investigations, an upper, low MDF, normal interval overlying a higher MDF reversed interval (higher in reversed samples containing no secondary normal components). Below a gap in core recovery is another reversed interval of similar stability, with slightly lower inclinations. In the rock magnetic measurements (Day et al., this volume), the division between the upper normal and reversed units is not very obvious, but a change in properties does seem to occur just below the top of the reversed unit (Table 1 in Day et al., this volume). The change in magnetic properties does, however, coincide with change in polarity (see also Site Report chapter, Site 407).

Between the two reversed units, in the lower part of this hole, a change in magnetic properties (Table 1 of Day et al.) seems to coincide with the change to lower inclinations, although all values fluctuate widely in this lowermost unit. Thermomagnetic data (Kobayashi et al., this volume) show a clearer delineation of the boundary between these two reversed units. Figure $3 \mathrm{a}$ in Kobayashi et al. (this volume) shows that below 420 meters, virtually all Curie curves are irreversible, and the average Curie temperature is higher than those of the upper reversed interval.

Not enough samples were examined in reflected light to see a difference between the two reversed units, but the upper normal and reversed units are clearly distinguishable by reflected light microscopy. Samples from the normal unit 
Curie Temperature, Microscopic Oxidation Stage

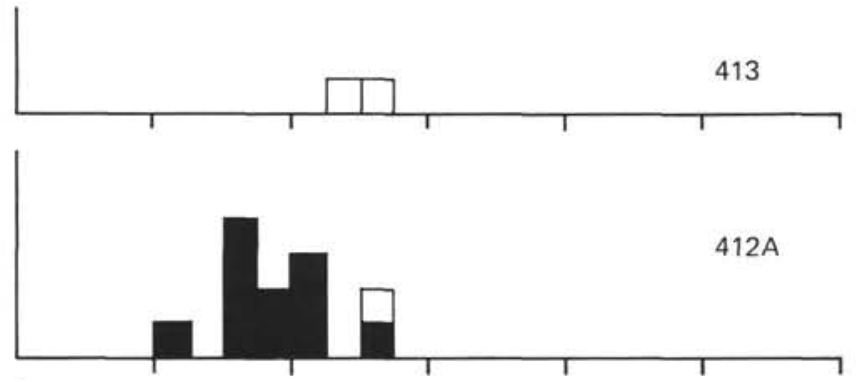

\lower unit

$?$

411
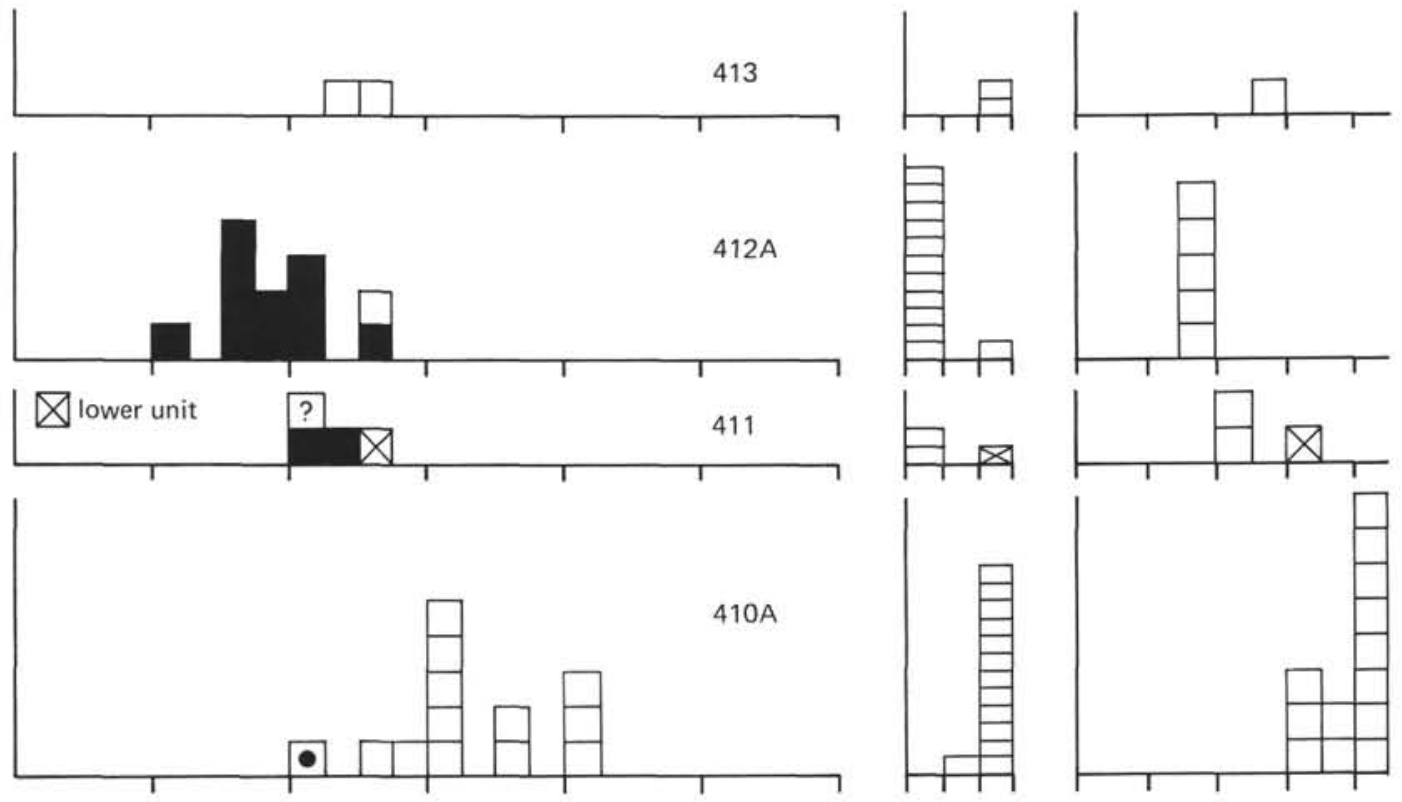

$\bigotimes$ small chunks

410
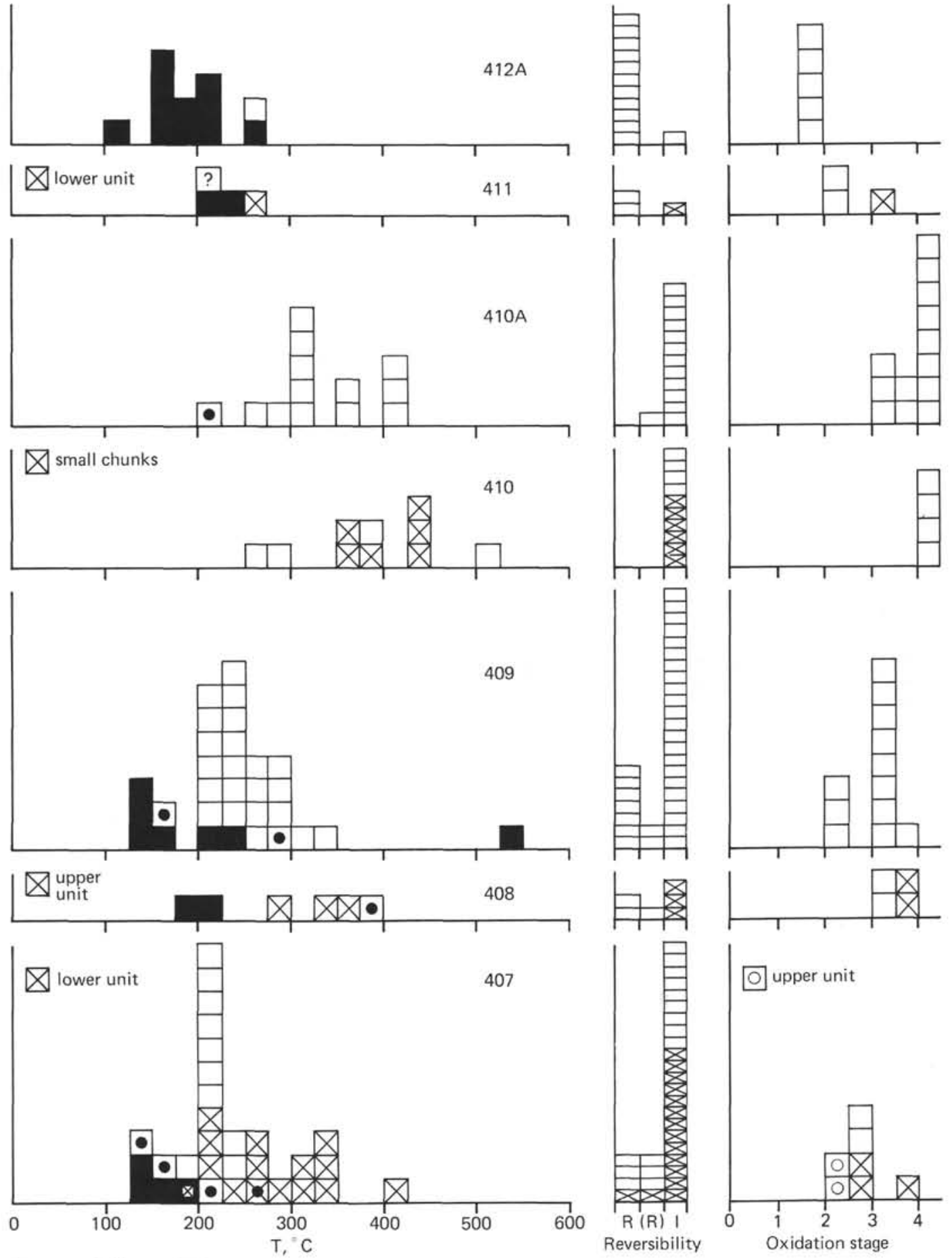

R $\bullet$ (R) $\square$ ।

Figure 1. Histogram of all initial Curie temperatures $\left(T_{c}\right)$ for Leg 49 samples; from Table 1 of Kobayashi et al. (this volume). Solid (open) squares denote reversibility (irreversibility); dots within squares are "partially reversible" (see Kobayashi et al., this volume); crosses denote various paleomagnetic units within each hole; open circles in the Oxidation Stage graph of Site 407 indicate the upper paleomagnetic unit. 

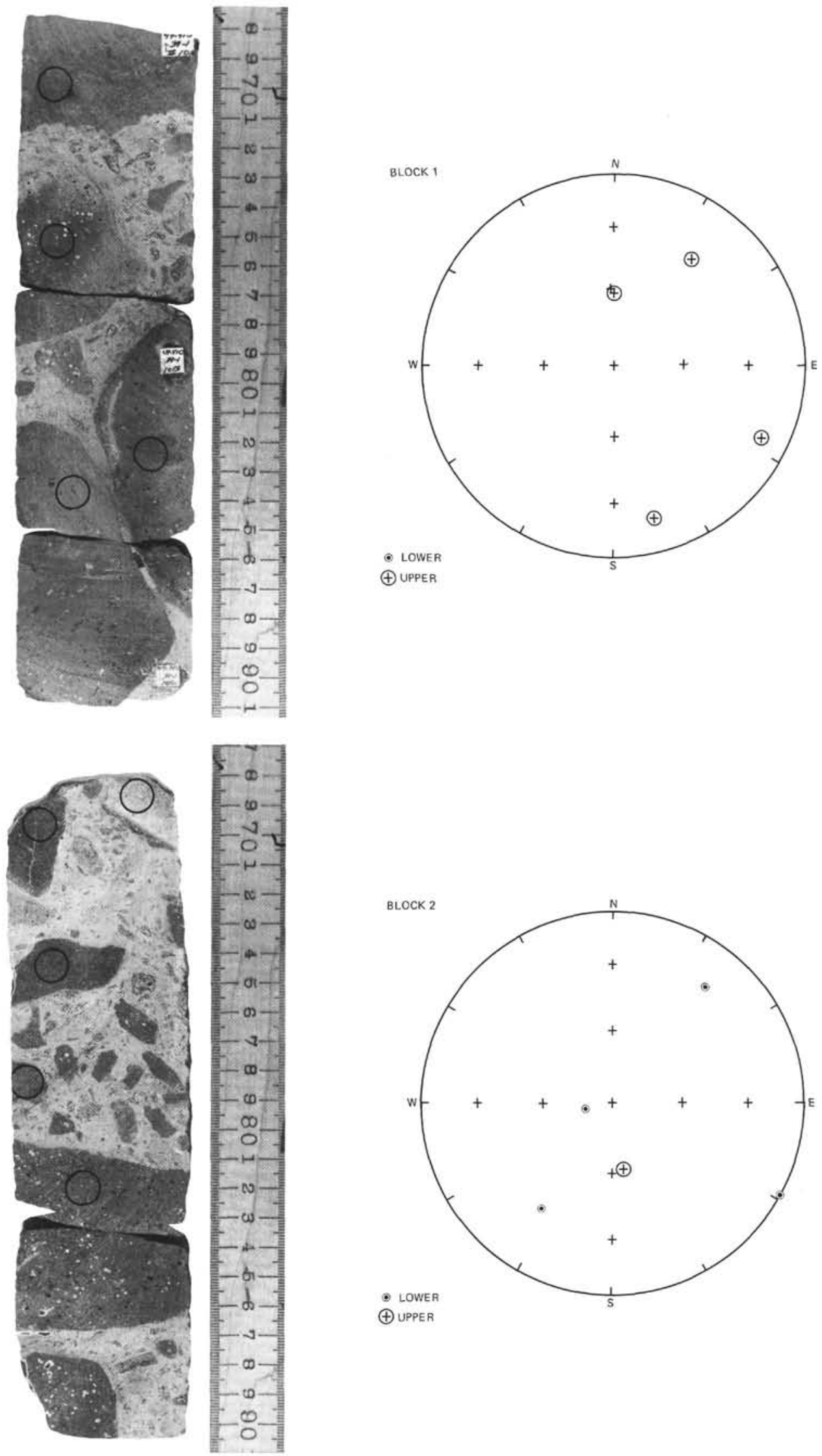

Figure 2. Small breccia pieces (Site 410) sampled for paleomagnetic study and their stable directions. Breccia Block $1=$ Pieces 10A, 10B, 10 C of Core 39, Section 1; Breccia Block $2=$ Pieces 10A, 10B of Core 39, Section 2. 


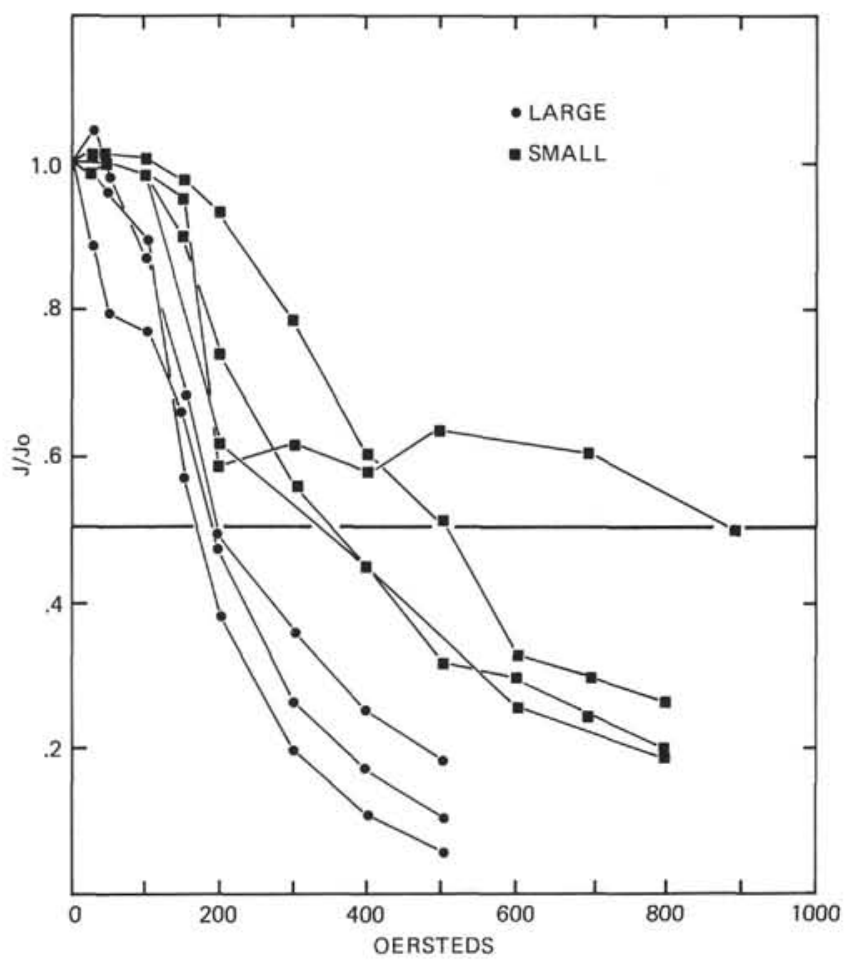

Figure 3. Comparison of remanence decay in response to alternating field demagnetization for typical large pieces and small pieces (Block 1 of Figure 2) of the breccia (Site 410).

appear to be very fresh (still containing brown magnetites), and the reversed units appear more c dized (Figure 1). Although Curie point data are sparse fo the normal unit, and do not indicate any great difference from the underlying reversed unit, the microscopic examination, the low coercivity, and high susceptibility suggest that this upper normal interval may be much younger (certainly much less oxidized) than the reversed intervals below it, implying that this may not be a record of a field reversal, but some other circumstance (perhaps off-axis vulcanism) that has caused a normal unit to overlie a reversed unit. Clearly, the data from Site 407 give confusing results: the upper normal unit appears to be less oxidized, but the polarity boundary does not coincide with the boundary in magnetic properties. No explanation can be advanced at this time, but it is interesting that this is also the site where the polarity of the crust does not correspond to that of the anomaly in which the site was so carefully positioned (see background section of Site 407 Site Report).

Hole 408 (20 m.y.) basalts also show a division, on the basis of paleomagnetic studies. Although only 40 meters were drilled at this site, a sharp change occurs midway through the section, a change in inclination, intensity, and MDF. This break is prominent in all rock magnetic properties listed in Table 1 of Day et al. (this volume). The change is from a more oxidized basalt above to a less oxidized one below, as was inferred from the visible alteration and the petrology of these basalts (see Site Summary Chapter).

The paleomagnetism findings (intensity, MDF, susceptibility) of Leg 49 are similar to other DSDP studies of basaltic crust (Legs 34, 37, and 45). Except for Site 407, there is agreement of the sign of the inclination at various sites with the magnetic anomalies as determined by towed magnetometer surveys. Inclination values are slightly too low, compared with the values expected for the latitudes at Sites 407, 408, 409 , and 411, suggesting that secular variation has not been averaged out at these sites. At all sites, many fewer magnetic units than petrographic units were identified, as would be the case for rapid or episodic extrusion relative to secular variation of the earth's magnetic field. It is noteworthy, however, that most inclinations are close to the expected inclination for each site, within the present range of secular variation, unlike the results of three of the five Leg 37 holes and results of Leg 46. Furthermore, the appreciable scatter in inclination values (Ryall et al., 1977) does not occur in Leg 49 holes. Scatter tends to increase with deeper penetration (Site 409), but still does not reach the values observed in Leg 37.

Very few high-susceptibility $(\chi)$ oceanic basalts, as those described by Lowrie (1973), Peirce et al. (1974), Johnson and Hall (1976), and Johnson (1976) were found; this may perhaps be attributed to the shallow penetration of Leg 49 drilling. Only a few samples from Hole 412A have high $\chi$. These samples are coarse-grained porphyritic basalts encountered periodically throughout Hole $412 \mathrm{~A}$. The generally coarser grain size of Hole 412A may account, in part, for its low stage of oxidation. Some of the basalt is described as cumulates, and shows evidence of crystal settling (Site Summary chapter). Site 412 has a mean inclination close to the inclination expected for that latitude. The implied longer crystallization time may be a reason for the lack of secular variation.

The relationship between degree of oxidation and grain size in Leg 49 basalts (Day et al., this volume) has been noted before. Johnson and Hall (1976) observed that the coarsegrained massive flow units $(40 \mathrm{~m} . \mathrm{y}$. old) had relatively unoxidized titanomagnetite grains, and all pillow basalts had been extensively oxidized at low temperature (see also Ryall et al., 1977). Our data certainly agree, since the most oxidized are the finest grained (Site 410 holes) and the least are the largest grained (Hole 412A). It would appear that grain size, which is related to mode of formation (pillow basalts or thin intrusions versus thick flows or intrusions), controls the degree and rapidity of oxidation among samiles.

Our data further suggest that the more oxidized samples of Leg 49 basalts contain single- (SD) and pseudo-singledomain (PSD) titanomagnetite grains, whereas less oxidized samples contain large pseudo-single and multi-domain (MD) grains. This is indicated by the $\mathrm{H}_{\mathrm{rc}} / \mathrm{H}_{\mathrm{c}}$ ratios (most samples have the SD-PSD values between 1.4 and 3), but is especially evident from the $\mathrm{J}_{\mathrm{r}} / \mathrm{J}_{\mathrm{s}}$ ratios, where most samples have values between 0.1 to greater than 0.5 (Day et al., this volume). This relationship is probably further indication that grain size and permeability of the rock controls the degree of oxidation, and that oxidation further diminishes the grain size (as suggested by Johnson and Hall, 1978).

\section{SUMMARY}

Leg 49 basalts are, in general, typical of oceanic basalts of previous studies. Their remanent intensity (NRM) is about 5 $\times 10^{-3} \mathrm{G}$, and shows no systematic variation with depth or age. Their inclinations are generally shallower than predicted 
by their latitude of formation, suggesting rapid extrusion of basalt, without averaging secular variation. However, inclinations of all holes are within the range of present secular variation. The abundant shallow and scattered inclinations observed on Legs 37 and 46 do not occur. The remanent magnetization is dominant in almost all cases: low $\chi$, high $Q$. In seven of eight holes, the sign of the inclination agrees with the magnetic anomaly.

All samples, even the youngest so far cored (0.95 m.y.), are low-temperature oxidized, in agreement with observations (Macdonald, 1977; Atwater and Johnson, 1977) that suggest that low-temperature oxidation, and associated lowering of remanence values, proceed very rapidly upon extrusion of the basalt. The degree of oxidation is, in general, low for all sites of Leg 49 except the Site 410 holes, where the tip end of pillowed, brecciated flows was apparently penetrated. The degree of low-temperature oxidation does not depend on either age or depth within the basaltic crust. It seems to be related to grain size and permeability of the basalts, which probably control access of hydrothermal solutions.

The unpredictability of oxidation stage is underscored by the fact that the youngest material (Site $411,1 \mathrm{~m} . \mathrm{y}$.) is more oxidized than the slightly older Hole 412A ( 2 m.y.). The freshness of Hole $412 \mathrm{~A}$ basalts is partially related to grain size, and may also be related to its position within a fracture zone, where it may have, at an early date, been sealed off from hydrothermal fluids.

Basalts of Leg 49, ranging from 1 m.y. to 40 m.y. old, have Curie temperatures between $123^{\circ}$ and $540^{\circ} \mathrm{C}$, generally between $170^{\circ}$ and $400^{\circ} \mathrm{C}$. They contain largely singledomain and pseudo-single-domain titanomagnetites, and have $x$ values (titanium content) averaging 0.66 . These values are generally nonsystematic in relationship to depth, age, or position relative to the Iceland hot spot. The range and average $x$ value agree with observations that oceanic basalts tend to form with $x \simeq 0.65$ (Johnson and Hall, in press). An apparent correlation of increasing Curie temperature and irreversibility with increasing $x$ value supports the hypothesis of removal of $\mathrm{Fe}$ from the titanomagnetite lattice as oxidation proceeds (Johnson and Hall, in press).

The basalts form an essentially bimodal population of more oxidized and less oxidized samples. The division shows up in the reversibility (irreversibility) of $\mathrm{T}_{\mathrm{c}}$ curves, higher or lower $T_{c}$, in microscopic oxidation stage, in coercivity (greater or less than $100 \mathrm{Oe}$ ), susceptibility, and saturation remanence. However, NRM intensity and stable paleomagnetic directions do not seem to be related to these properties. Within a paleomagnetic unit, within a site, NRM values are higher in less oxidized samples, but generally still within an order of magnitude, and inclinations are the same in more and less oxidized samples. These observations support the idea of fast initial oxidation, decreasing the remanence, possibly before the earth's field direction changes, and gradual, continuing oxidation that continues to slowly harden the remanence, raising the Curie temperature, raising the coercivity, lowering the susceptibility, and slowly further lowering the value of the natural $(\mathrm{NRM})$ and saturation $\left(\mathrm{J}_{\mathrm{s}}\right.$, $\left.\mathrm{J}_{\mathrm{rs}}\right)$ remanence.

\section{ACKNOWLEDGMENTS}

We are grateful to Paul Johnson for discussions review of the manuscript.

\section{REFERENCES}

Irving, E., 1970. The Mid-Atlantic Ridge at $45^{\circ} \mathrm{N}$, XIVOxidation and magnetic properties of basalt, review and discussion, Canadian J. Earth Sci., v. 7, p. 1528-1538.

Johnson, H.P., 1976. Magnetic properties of crustal rocks from the Mid-Atlantic Ridge at $23^{\circ} \mathrm{N}-$ DSDP Leg 45, Trans. Am. Geophys. Union, v. 57, p. 932.

Johnson, H.P. and Atwater, T., 1977. Magnetic study of basalts from the Mid-Atlantic Ridge, lat $37^{\circ} \mathrm{N}$, Geol. Soc. Am. Bull., v. 88 , p. $637-647$.

Johnson, H.P. and Hall, J.M., 1976. Magnetic properties of the Oceanic Crust: Considerations from the Results of DSDP Leg 34, J. Geophys. Res., v. 81, p. 5281-5293.

, in press. A detailed rock magnetic and opaque mineralogy study of the basalts from the Nazca Plate, Geophys. J. Roy. Astron. Soc.

Lowrie, W., 1973. Viscous remanent magnetization in oceanic basalts, Nature, v. 243 , p. 27-30.

Macdonald, K.C., 1977. Near-bottom magnetic anomalies, asymmetric spreading, oblique spreading, and tectonics of the MidAtlantic Ridge near lat $37^{\circ} \mathrm{N}$, Geol. Soc. Am. Bull., v. 88, p. 541-555.

Peirce, J.W., Denham, C.R., and Luyendyk, B.P., 1974. Paleomagnetic results of basalt samples from DSDP Leg 26, Southern Indian Ocean. In Hart, S.R., Yeats, R.S., et al. Initial Reports of the Deep Sea Drilling Project, v. 34: Washington (U.S. Government Printing Office), p. 517-527.

Ryall, P.J., Hall, J.M., Clark, J., and Milligan, T., 1977. Magnetization of oceanic crustal layer 2 - results and thoughts after DSDP Leg 37, Canadian J. Earth Sci., v. 14, p. 684-706.

Schilling, J.G., 1973. Iceland mantle plume: geochemical study of Reykjanes Ridge, Nature, v. 242 , p. $565-571$. 